gether in a "closed" position being one-half inch, which is the thickness of a printing plate, and covering an area equal to the length and breadth of a newspaper page. At one of the straight sides of this aperture is
arranged a nozzle, which connects by means of a throat with a pump immersed in a pot containing about 12,000 with a pump immersed in a pot containing about 12,000 pounds of stereotype metal. The back of the casting chamber, which is arranged to rise and fall, in its unskilled men, but it will suffice to say that the Junior autoplate is substantially "fool-proof," as was evi denced at the convention, when a number of news paper men endeavored in different ways but without success to put it out of order.

Junior autoplates have been purchased by the Brooklyn Daily Eagle, the New York Evening Post, the BufTrening News, the Boston Traveller, the Boston Daily Globe, the Philadelphia Inquirer, the Philadel-

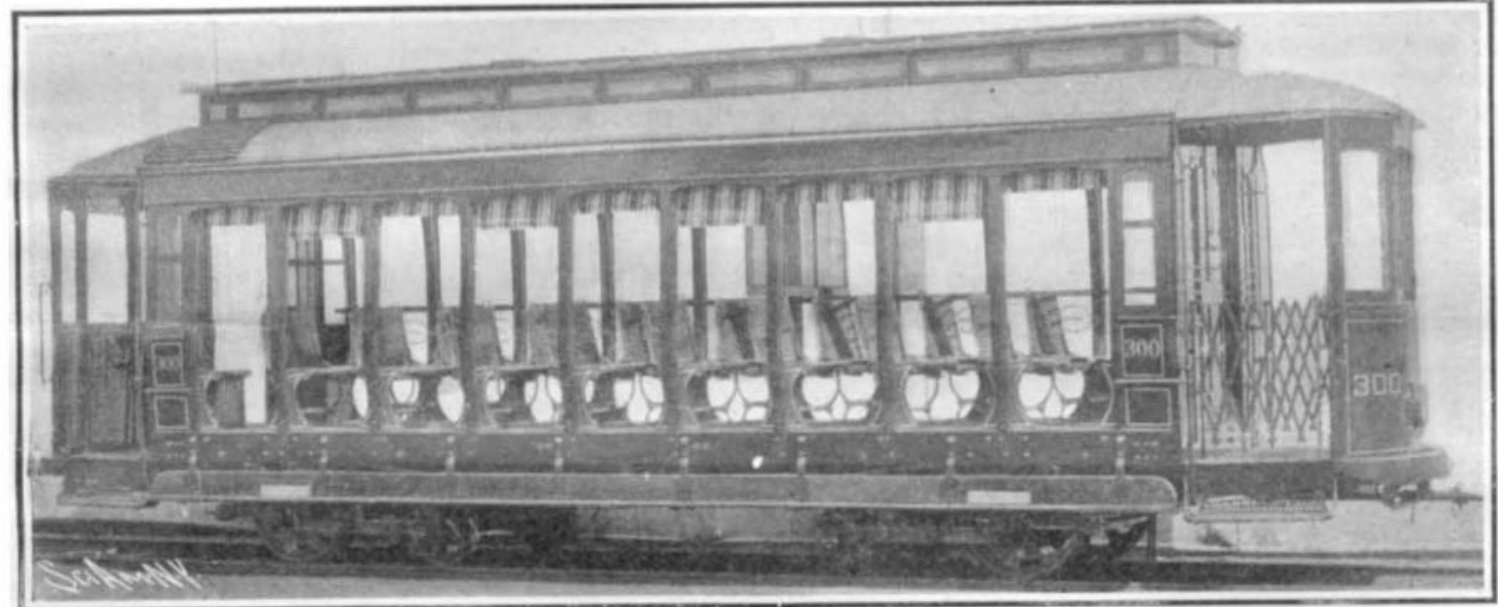

THE NEIV CONVERTIBLE CAR.

permit of the insertion of the papier-maché mold. A description of the operation of the machine will perhaps sufficiently describe its mechanism. The mold having been set in place, a lever starts the apparatus to work and the back rises, bringing the mold into position with respect to the core, when the pump in. position with respect to the core, when the pump in-
jects the necessary amount of metal, which is in the neighborhood of fifty pounds. The pump pressure is continued for ten seconds, during which the cooling of continued for ten seconds, during which the cooling of the cast is effected by means of a spray playing within the shell of the core and a circulation of water within the back. The cast having solidified, the back drops away and carries with it the matrix, which by means of this motion is stripped from the printing surface of the plate, that is left adhering to the underside of the core. The core then makes half a revolution and brings the printing plate uppermost, while the back again closes upon the underside of the core, and another plate is made. Carrying mechanisms meanwhile remove the mist plate frot the convex side uppermost, through a series of finishing devices which rent its un erly bevel it for the press clamps. The work is so pererly bevel it for the press clamps. The work is so perfectly dome brought to an exact thickness, so that it will properly fit a printing cylinder.

fit a printing cylinder.
Thus, by means of this machine and three men, printing plates may be made at the rate of four or five a minute, whereas before its introduction from eighteen to twenty men were required for the same work, and but one plate a minute of each page was obtain-

In his latest invention, the Junior autoplate, $\mathrm{Mr}$. Wood has employed the rotating core and the reciprocating back of his larger machine, but has stood the casting parts on end, so that the axis of the cylinder casting parts on end, so that the axis of the cylinder
is vertical, and the casting chamber is filled, as the illustrations will show, by a hand pump, which with one stroke delivers sufficient metal for a plate. In one stroke delivers sufficient metal for a plate. In
this device the casting chamber is closed and opened by means of a hand lever, but the mold as in the autoplate is mechanically stripped from one cast and repositioned for a succeeding one.

In working the Junior, the operator having closed In working the Junior, the operator having closed
the chamber and filled it with metal must await the cooling of the cast, and here, in order that the workman may know precisely when to remove it, Mr. Wood assists him with a mechanism which, once set for the time necessary for cooling, will always strike a gong when the specified time has elapsed. Then the op erator quickly opens the casting chamber, sets a clutch which starts the cylindrical core, driven by power, to make half a revoution, and the cast is delivered from the chamber while, during the turning operation, two saws having been brought into play, one separates the cast plate from its riser and the other makes a bevel upon its lower end

upon its lower end.
When the cylinder comes to rest, the chamber is again closed and the operator pours his metal for a succeeding cast, but in its closing the back of the chamber thrusts the plate and its riser away from the chlinder, so that, while waiting for the next bell, the cylinger soy remove the plate, drop it into a sm, the operator may remove the plate, drop it into a smoothsary for finishing, and may lift off the riser to toss it sary for finishing, and into the metal pot.

By means of this simple apparatus, which is semiBy means of this simple apparatus, which is semitelligence upon the part of those who run it, three plates a minute may easily be produced by two men. phus it will be seen that Mr. Wood has gone still further in the direction of saving labor in stereotyping, and seems now to have completed the revolution in newspaper stereotyping which he began with the autonewsp
plate.

Space does not permit us to describe in detail the ingenious safety system which $\mathrm{Mr}$. Wood has employed to prevent mishaps to its mechanism when handled by phia Bulletin, the Milwaukee Journal, the Minneapolis Journal, and many other papers.

NEW SEATING ARRANGEMENT FOR STREET CARS.*

By GEORGE J. JoNES.

As innovation in the equipment of street railwa cars has been recently turned out by the J. G. Brill Company, of Philadelphia, $\mathrm{Pa}$., which is shown in the accompanying cuts. It was originally designed to meet the exigencies of the service in the Philippine Islands, where the first cars of this character were shipped and are now in operation. The new idea has been found to be such an excellent one that it is probable that cars of this description will soon be seen in the cities of this country. The rolling stock is of the convertible type, and when the sides are closed, as in cold or rainy weather, it is essential that there should be an aisle down the center of the vehicle so that passengers may make their way to and from the seats. In the warm weather, when the sides of these cars are disposed of by raising into pockets in the framework of the roof, the presence of the aisle is unnecessary and it is desirable that it should be given over to the accommodation of passengers, For this purpose a "filler seat" has been designed which is hung in the passageway between the two side seats.

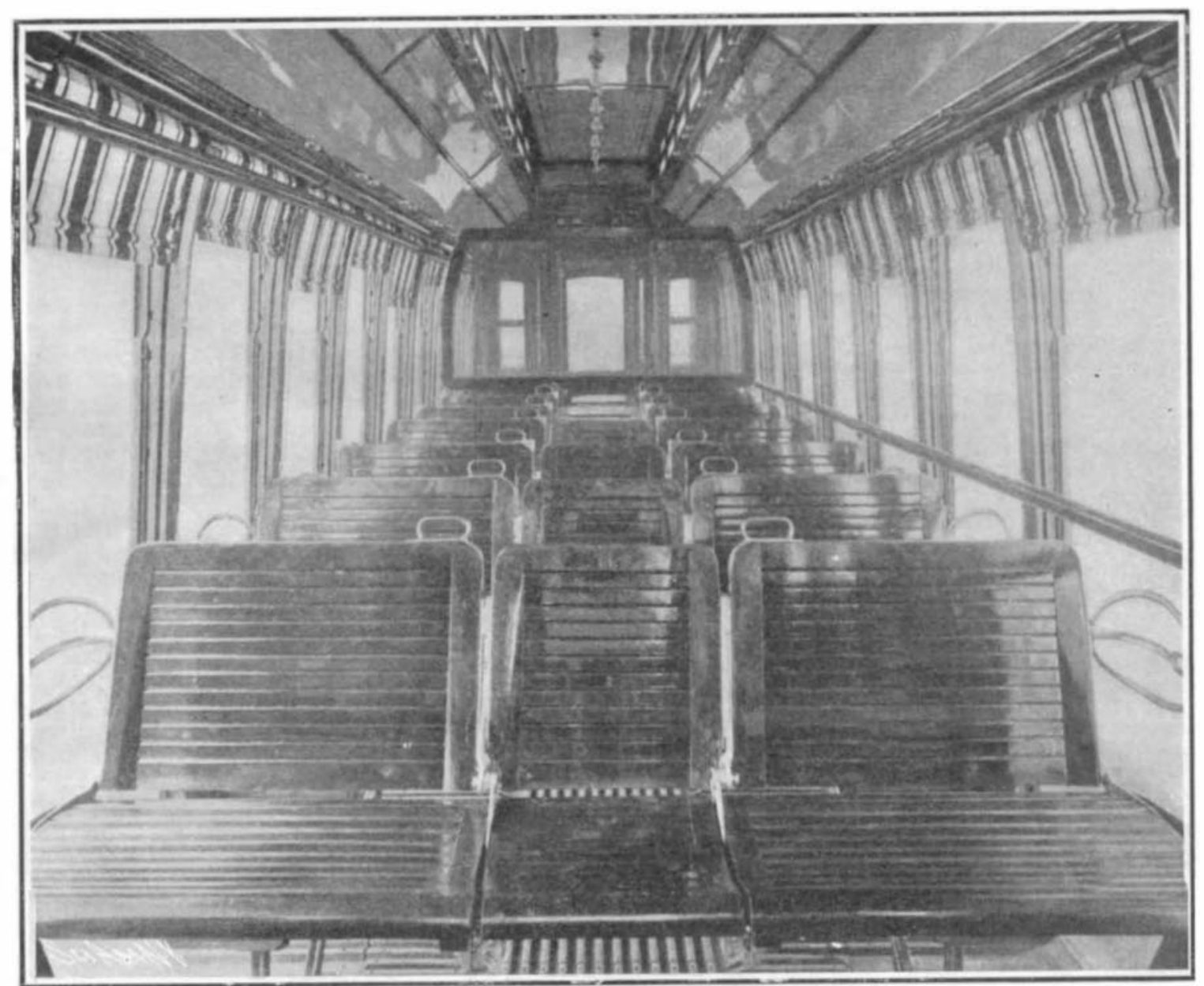

INTERIOR OF CAR WITH AISLE SEATS IN POSITION.

In this manner one full-size seat is presented extending across the entire width of the vehicle. The change is made twice each year, and when not in use the fille seats are stored in the barn.

A feature of the construction of this car for service in the Far East, and one which is not likely to be demanded in this country, is the screen which is to be seen dividing the interior of the vehicle into tw compartments. The social conditions existing in the tition is of fender this necessary. The dividing partition is of fine wire, which accomplose have a survey of the interior at all times.

CONCRETE MIXING MACHINERY.

By William L. Larkin.

TIIE mixing of concrete by means of machinery is of recent date. The first machine which suggested itsel to contractors was the old style "roller and pan" mill used in grinding clay. Robert Hunt, in his "Dictionary of Arts, Manufactures, and Mines," of 1875, speaks of mixing concrete both by hand and by machine. He says that the best mode of mixing is to take burn limestone with ballast (i. e., gravel) in a dry state and after adding sufficient water turn with shovels and wheel away. He states that in placing concrete in the forms it should be given a fall of some yards to drive the particles closer together and give the whole a greater solidity. In this way he-curiously enough - touches upon one of the principles that are strongly advocated by some makers of concrete-mixing machinery at the present time, that is, a violent mixing of the aggregates and cement. He goes on and says that in some cases it has been found advantageous to mix the ingredients in a "pug" mill, probably meaning the old style of pug mill used in brick-making establishments, in which there were blades intended to mix, and also to break up large lumps of clay. Knight's "American Dictionary" of 1882 speaks of concrete mixing in pug mill. John Newman, in his "Notes on Concrete" of 1887, speaks of an apparatus at Newhaven, England, capable of automatically measuring, mixing, and de livering 70 yards of mixed concrete per hour. He also speaks of the pan and roller mill like that used for grinding clay, and suggests that a "trough paddle mixer" or "pug mill," preferably

W. M. Patten's "Treatise on Engineering," of 1895, speaks of a "cubical box" and a "trough" mixer as be ing the most common, as they were at that time. Since 1875 the making of concrete mixing machiner has had a rapid growth. Contractors soon found that the "Cube mixer was not convenient enough, and that the "trough" mixer was neither good enough nor durable enough. Like all new fields of effort it was soon overrun, and to-day there are many makers of concrete mixing machinery and many different kinds of machines. The principle on which concrete machinery is built may be outlined briefly here. In the first place, it is desired to turn the material as many times as possible in a given period; secondly, it is most important that the material be thrown from side to side so that the resulting mixture may be perfectly homogeneous. To enlarge upon this point, which is one of the most important ones, imagine for a moment he concrete mixer is in ually pertain on a job wher 
to drop in the mixer close to one side. Thus while the stone and sand are placed in the body of the mixer more or less evenly spread around, the cement is
almost invariably dropped in at one point. Should almost invariably dropped in at one point. Should the mixer not be designed properly the resulting mix ture would be very rich in cement on one side of the drum and very poor on the opposite side. As a preventive of this defect means are placed in the mix ing drum to cause the material on one side to be thrown clear across to the opposite side, and vice versa, so that when the mass is finally mixed it is
thoroughly homogeneous and as well mixed in one place as another.

It is beginning to be understood that the more violently the aggregates are thrown together and tumbled about the more thoroughly they are mixed and the better they become coated with cement. It is the object of many makers of modern machinery to violently mix the aggregates and cement by lifting them high several machines are designed with what are called "coating tables" or stationary plates in the interior of the rotating drum upon which the aggregates are violently thrown, tending to grind the mass together Another defect to be found in the earlier machines was the tendency of the mass to "ball up" as snow will form into a snowball when rolling down the side of a hill. In fact, the old cubical box type of mixer which particularly. has this tendency is used at the present It may be said that the rough usage makes it necessary that concrete machinery be designed in a thoroughly strong and rigid manner, and that all parts subject to wear be made so that they can be renewed easily and so that they will resist abrasion as far as possible. It is well known that cement dust and grit bearing boxes, and for that reason boxes which support the journals of concrete mixing machinery not only should be dustproof but they should be self-oiling, as it is obvious that the breaking down of a $\$ 2$ journal box on a concrete mixer might easily cause a loss of as many hundred dollars to the contractor by the stoppage of his work. All wearing surfaces like roller
wheels and tracks for the same, should be made of possible should be left unfinished so that the hard scale may remain and leave an excellent wearing surface. Concrete mixing machinery should be of ample capacity to hold the material without spilling or slopping as far as possible. In conclusion, it may be said that the designing of concrete machinery should follow words, nearly all cement is sent to contractors in bags which contain about a cubic foot of material. Batches of aggregates and cement to be mixed are usually laid out in what are called 1-bag batches. 2-bag batches, or 3-bag batches; that is, the size of the batch is based sists of 1 bag. The 1-bag batch of 1-3-6 concrete conent, 3 cubic feet of sand, and 6 cubic feet of stone; about 2 cubic feet of cement, 6 cubic feet of sand, and 12 cubic feet of stone; and the 3-bag batch consists of 3 bags of cement, i. e., 3 cubic feet of cement, 9
cubic feet of sand, and 18 cubic feet of stone. By adding up the number of cubic feet in each batch it will be seen that the 1-bag batch consists of 10 cubic feet loose aggregates, the 2-bag batch consists of 20 cubic feet of loose aggregates, and the 3-bag batch consist
of 30 cubic feet of loose aggregates.

which the greatest amount of stone is used as pared with cement. For that reason many concrete mixing machinery makers have rated the capacity of cubic-feet machines; in other words, machines that will hold a 1-bag batch, a 2-bag batch, and a 3-bag batch. In view of present practice this seems to be the most ing machinery.

There are in general use at the present time two great classifications of concrete mixing machinery. ers, and the second ous" mixers. The batch mixer class includes machines such as the cube mixer, the cheese-box style, the tipping mixer, and several others. The continuous class or two rotary cylindrical mixers, and the portable gravity mixer. The distinction between these two for which a certain measured quantity of concrete is laid out, introduced into the drum, mixed, and discharged. When this operation is completed the sec-
ond batch or quantity is measured out and put through the same course. The continuous type of mixer is one the same course. The continuous type of mixer is one
in which mixing is always going on. It is continually receiving portions of fresh batches and continually discharging mixed portions of batches previously in
troduced. In other words, it mixes and discharges troduced. In other words, it mixes and discharges kinds of concrete mixers at the present time on the
market, some of which are popular from long service some of which may be said to be popular in certain geographical districts, some of which are popular bemake them either labor-saving or more durable for the work.

As said previously, the cubical box mixer is one of the oldest styles of mixer still in use. Generally consists of a large hollow cube of sheet steel turning on a horizontal axis through two of its corners. trap door is placed on one side of the cube at some
convenient point and the whole mounted on a high tower with proper means for rotation. This tower is usually built of sufficient height and strength to support a storage bin for the stone, sand, and cement be-
fore the same are mixed, and to allow teams, cars, etc. to run under it and receive the mixture when it is disharged. The cubical box mixer is used to-day o many large jobs. This old style cube mixer at the present time is manufactured by all large makers of Machinery Company of New York recently brough out an improvement on the old style cube mixer by making a circular opening at one of the corners through which the axis passes through which the batch may be introduced without stopping the rotation of the mixer. This adds to the value of this type the other good points of the cubical box.

The next style of mixer which came into general style, of which the "Ransome," Gilbreth's "Rotary," United Concrete Machinery Company's "Gotham," "American," the "McKelvey," and "National" are ex amples. This type of machine generally consists of a cylindrical drum with an opening in each head, one for introducing the charge or batch, the other for removing the batch after it is mixed. These mixers gerierally contain some type of blades attached to the inner surface of the circumference in such a way that they will throw the mass from side to side, lift it up into the air and throw it down, and finally chute it into the discharging means. Some of them depend on the introduction of an iron chute into the discharge hole which catches the falling material on the inside of the drum and discharges it to the exterior by means mixer unique in that the opening in each head of the cylinder is large enough so that a wheelbarrow guided by certain fixed iron supports may be introduced entire into the mixer. It is also unique in that it may be first apparatus which introduced the feature of stationary coating tables on which the aggregates and cement were violently thrown and mixed and ground together. The United Concrete Machinery Company's "Gotham" seems to be unique in that it is the first rotary concrete mixer on which were placed dust-proof bearings. One of the most interesting types of concrete mixing machinery is that which is known as the "tipping" style of mixer, of which the Smith mixer, the Municipal Engineering and Contracting Company's mixer, and the United Concrete Machinery Company's "United" mixer are the most prominent examples. These able supports, and provided with openings at either end, one for receiving the charge and one through Which the charge may be thrown out of the mixer frames are so arranged that when the charge is entirely mixed the drum is tilted in such a manner that the mixed concrete runs out the discharging end by gravity alone. The Smith mixer takes the shape of a small shelves which are arranged to throw the material back and forth. The tipping mixer of the Municipal Engineering and Contracting Company consists of a cube mounted in such a manner that it can be vertices; it has no blades on the inside. The "United" tipping mixer of the United Concrete Machinery Company combines the good qualities of the cubical box machine with the large capacity of the cylindrical which is formed a pyramidal discharging end. Inside the drum are placed radial blades which lift the material high in the interior of the drum and cast it down again upon a stationary coating table, which
tends to grind the material together. The back and forth motion in the inside of the "United" drum is obtained by means of the inclined faces of the pyramidal-shaped end, and by the inclined position of this coating table. While other types of tipping mixers with a steam-actuated tilting apparatus of very simple and efficient design, which is a means of saving time. This mixer has another interesting point, namely, that the engine which rotates the drum is mounted on the frame, obviating the necessity of a bevel gear connection between the tipping frame and the usual stationary engine. The engine used on the "United" mixer ple in design and so mounted on the frame that it can be directly connected to the drum by means of spur gears. It is so placed that the maximum of work upon the mass of aggregates and cement, making it possible to get a better and more thorough mix in a shorter time.

Another type of mixer is known as the "Trough" ing Company, the Pioneer Iron Works, and many others. This trough style of mixer is one of the oldin clay working plants. $l$ generally consists of a long horizontal trough oper at the top through which shaft upon which are mounted a. series of iron padshaft upon which are mounced a. series of iron pad-
dles which take the shape of a screw-propeller of a and the batch of concrete shoveled into the mixer, the paddles tending to mix the material and shove it toward the discharging end. The trough is usually placed at a slight inclination to aid the thrust of the paddles in causing the material to run out at the lower end into wheelbarrows or other receptacles placed for it. Another style of concrete mixer which is more or less unique is what is known as "Portable Gravity" concrete mixer, invented some years since wrank B. Gilbreth, the well-known contractor. Then he present time. Howerher waving a piece of work which required mixed concrete in the foundation, it occurred to him that it might be a good idea to set up an inclined chute from the street level down to be placed on end in a vertical position a lot of large spikes or small iron rods. This was done and when the batch on the street level was laid out the laborers shoveled it into this chute or trough in the hope that by the time it reached the bottom it would have been
sufficiently tangled up by contact with the pins, etc. in the trough to have received more or less of a mixfrom this crude idea the first portable gravity mixer was developed, which since that time has had a worldwide sale, having been used in the building of fortifications in Japan, Great Docks of Liverpool, public works of South Africa, great battery emplacemen
the United States, and innumerable other jobs.

METHOD OF OPERATING CONCRETE MIXERS.

The methods of operating concrete mixers are of
course as varied almost as the number of works on which they are used, but the machine is usually placed conveniently to a raised platform on which the batch is laid out, measured, and from which it is shoveled derrick the same platform is used for the convenfence of the man who handles the derrick boxes. The mixer is so placed that the discharge end is sufficiently high to allow the matter to be chuted out into such condump buckets. It is evident that it is a desirable thing to have a mixer whose charging opening is as low as possible and whose discharging opening is as high as possible, so that the platform may be as near itself may level as possible, and so that the mixer ground to discharge. The usual method of operation is to place two gangs of measurers and shovelers on them to work which is built of sufficient size to of the mixer. Two other gangs are usually employed with wheelbarrows or other means in bringing stock from the stock pile to the platform. Each gang of measurers and shovelers is provided with a low-sided bottomless box so designed that when it rests on the of cubic feet require it will exactly hold the number amount of stone is dumped into this bottomless box and gauged by a mark on the inside surface, after which the necessary sand is placed over it, and finally the cement spread upon the sand. When this has been done this bottomless box is lifted from the platcement, which is then shoveled into the mixer. Two gangs of men are employed on the platform so that while one batch is being shoveled into the machine another may be measured. This is perhaps the simplest and crudest way of feeding concrete mixers, the next better scheme being that of measuring into scale
boxes of sufficient size to hold the required batch, and which are handled by derricks. These scale boxes are dropped near the stock piles and there loaded by the men, four or five gangs usually operating at the same up by the derrick and emptied into the mixer. Another scheme commonly used consists of attaching to the mixer a large iron hopper of the necessary size to may the batch, in which the aggregates and cement After one batch has been discharged the second batch is rapidly introduced into the drum from the hopper by means of a door or gate.

As previously remarked, there are many different styles and types of concrete mixing machines; but in the last twenty-five years a large amount of experience has been gained with the result that the best practice in mixer design has come down to one or two standard shapes and kinds; and it should be the object of prospective purchasers of concrete machinery to avoid what might be called freak mixers, using just discrimination in selecting that machine of standard type which comes nearest to suiting their needs.

On very large jobs where the material is conveyed to the mixer by overhead tram road or by bucket eleor other removed after having been mixed by cars The portable, the cube mixer has considerable favor. sort of feeding device is also being used for similar work; and in fact probably the largest piece of concrete construction in the world at present under way is Hills, near Sheffield, England. There concrete is being mixed by portable gravity mixers attached to what large jobs where the concrete is handled mostly by derricks, the cheese-box or cylindrical type of batch mixer and the tipping mixer are probably used in 90 per cent of the cases; and this classification of large
jobs where the material is handled by the derrick crete is used. The main reason for the use of these 
two types is that they are the most convenient to se up and handle and that they may be shifted from on part of the job to another without much trouble. On abutments, etc., it might be said that all kinds rotary mixers are in general use. For street paving jobs the portable trough or paddle mixer, some type of cylindrical drum continuous mixers, are quite commonly used, for the reason that they are very conveniently mounted on wheels, have low charge and discharge openings and are convenient to operate crete block makers often call for hand power mixing machinery.

FEEDING DEVICES FOR CONCRETE MIXERS.

After concrete mixing machinery had been prett well developed attention was turned to the questio of devices for feeding the aggregates and cement int the mixer. As has previously been explained, these
devices first took the form of hoppers attached to the devices first took the form of hoppers attached to the mixer, large platforms from which tip carts could be dumped direct Into the apparatus, belt elevators feed ing into the storage bins, inclined track hoppers, as cxamnled bv the "International" mixer of the United Concrete Machinery Company, the portable set-up as
used in connection with the portable gravity mixer used in connection with the portable gravit
etc.

With the increasing use of concrete machinery it became evident, particularly on very large works, that some means of measuring the aggregates to be fed into the concrete mixer were necessary other than that of hand labor, partly on account of the expense, partly on account of-the fact that hand work is never as accurate as machine work. As long as cement costs money it will be the desire of cement users to put the right amount of cement in the concrete and no more. It is the engineer's desire that the concrete be absolutely uniform in strength, not being stronger in one place than in another. This defect is quite possible where one man measures the cement for a week and then is substituted by another man who may be less accurate than the previous man. Again, it is pretty certain that in a single day's work the conorete will vary in
strength from hour to hour, because of the fact that the gang loading stone and sand into the scale box shovel a little more one time and a little less the next With the end in view of making concrete more homogeneous, of saving labor, and avoiding inaccuracy, a number of concrete machinery makers have put out what are known as "Measuring and Feeding Devices." Of these devices might be mentioned the "Autometric" mixer, manufactured by the Bonnot Company. It consists of a large cylindrical drum shaped more or necessary blades for mixing the concrete when the necessary blades for mixing the concrete when the
cylinder is rotated. One end of this apparatus rotates cylinder is rotated. One end of this apparatus rotates
in a bin or box with compartments for sand, stone in a bin or box with compartments for sand, stone,
and cement. On the outside of the drum are peculia pockets which pick up stone from the stone bin, san from the sand bin, and cement from the cement bin, and carry them around to a certain point where they automatically slide into the body of the mixer. The Foote mixer is an example of small measuring and able for road work and small jobs. The Connolly and able for road work and small jobs. The Connolly and
Fenn mixers are provided with belt elevators which pick up material from bins placed at a low level. The elevator consists of three parts; one belt carrying a pick un ner of buckets placed close together which pick up the stone, the second belt carrying one-hal
as many buckets which pick up the sand the third belt carrying a variable number of buckets, say onethird as many as there are on the sand conveyer which serve to pick up the cement. In other words the proportions are obtained by varying the number o the stone, sand, and cement into a receiving hopper a the stone, sand, and cement into a receiving hopper a down to the mixer itself, which usually takes the form of a trough mixer.

One of the most successful machines for measuring and feeding is known as the "Gilbreth Accurate Measurer and Feeder," an apparatus particularly suitable for very large jobs. It consists of a tower usually is a large storage bin ccentaining three compartments. one for sand, one for stone, and one for cement. Each of these compartments terminates in a small iron chute whose front side is formed by a hinged and weighted gate. The bottom of each chute is formed by the surface of a large drum directly underneath
this drum being supported in the tower in such this drum being supported in the tower in such a manner that it may be rotated under the chutes by means of hand power. The method of operating is as derricks, tramways, elevators, or other means at hand, derricks, tramways, elevators, or other means at hand,
and the mass in each compartment rests on the drum itself through the chute underneath. These chutes and the drum are so designed that when the latter is the drum is rotated the material begins to roll off the drum and pass down through the gates at the front of the chutes. The aggregates and cement as they fall "concentrating" hopper and are guided into the mixer "concentrating" hopper and are guided into the mixer preferably the "Portable Gravity" type. It is evident that the amounts of stone, sand, and cement whic pe proportionate to the areas of the openings. By adjusting these gates or openings the desired proportions to the speed at which the drum is turned. This apparatus has been found very useful on large jobs, and its
accuracy is easily within $1 / 2$ of 1 per cent.

In conclusion, it might not be out of place to sug gest to those to whom the subject of concrete machinery is not familiar the methods by which they may and the qualifications which a mixer should have. In the first place, good concrete is that which has sand enough to fill all voids in the stone, cement enough to fill all the voids in the sand. It is generally considered that on the mortar, that is, the sand and cement, depends the strength of the final mass. It may be generally said that the amount of mortar should be always in excess of that required to actually fill the voids in the stone. Concrete properly mixed may be known in a number of ways-perhaps the best and easiest test being that of color. Where a mass of concrete shows a variation in color it is pretty certain to be poorly mixed, being reddish in spots, due to the color of the sand, while on the other hand properlymixed concrete is an even gray color showing that all
the stones and grains of sand are perfectly coated with cement.

A stony appearance of the resulting mass indicates too little sand. It is very difficult to decide on the samples of the actual stone and sand to be used are inspected and measured.

The question of the amount of water to be used in the mixing of concrete has been referred to previously in this article, and it may be said. that care should be taken that in the handling of concrete too much water is not added so that the cement will be washed away from the stone and sand in places. This is especially true where the resulting mixture is to be

quantities, as by the wheelbarrow load.

In conclusion, it may be said that defects in mixilas they are sometimes remedied in handling where the mass is dumped in large quantities from cars or scale boxes into a dam or some large structure, as in such a case the very act of dumping causes the stuff which is gamated with the rest of the mass.

\section{A Simple method of measuring the} DENSITY OF LIQUIDS.

THE only special instruments required for the meas urement of the density of a liquid, to the second place

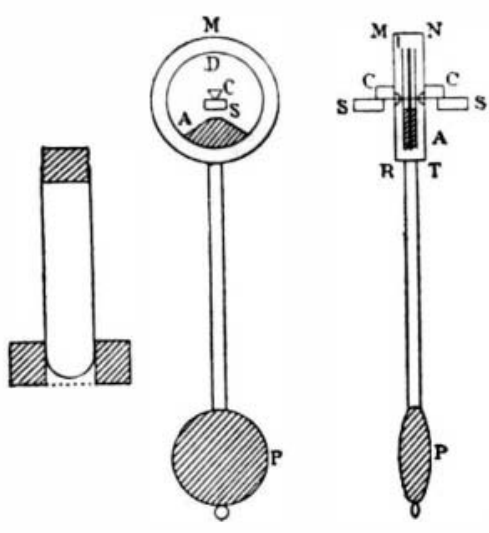

of decimals, are: 1. A small balance (coin balance) sensitive to 5 milligrammes, which may be purchased [in Paris] for 15 francs (\$3). 2. An accurately
marked double pipette, of 10 cubic centimeters' pacity, which will cost about a franc and a half (30 ents

In addition there is required a receiver, consisting of a test tube about five inches long, mounted on a in the illustration.

The method of operation is as follows: First, the test tube is thoroughly dried. This may be done quickly and easily by heating it in the fiame of a Bunsen burner. When cool, the tube with its cork and base is placed in one pan of the balance, and a counterpoise of about 30 grammes is put in the othe added to the first pan until the pointer of the balance returns to the zero mark. Calling the sum of these small weights $\boldsymbol{P}_{1}$, we have, from the first weighing: Counterpoise $=$ receiver $+P$

Next, 10 cubic centimeters of the liquid whose density is desired are drawn into the pipette and transferred to the receiver, which is re-corked and replaced equilibrium pan. Calling

Counterpoise $=$ receiver +10 c.c. of the liquid $+P_{\%}$.

The test-tube is then emptied, thoroughly washed, and dried. Ten cubic centimeters of distilled water is placed in it with the aid of the pipette, and it is replaced, corked, on the same scale pan, with weights sufficient to establish equilibrium.

Calling these weights $\boldsymbol{P}_{3}$, we have Counterpoise $=$ rece

Hence, weight of 10 c.c. liquid $=P_{1}-P_{2}$ and density of the liquid $=\frac{P_{1}-P_{2}}{P_{1}-P_{3}}$

At the temperature of my laboratory, $12 \mathrm{deg}$. C. (54 deg. F.), $P_{2}-P_{3}$ will be found equal to 10 grammes weights are correct. As an example, the density found
for bisulphide of carbon by this method is $\frac{12.645}{10}=$ 1.264. The density given by the annual of the Bureau ran in La Science au XXme Siècle.

\section{THE N-RAY PROBLEM}

As interesting contribution to the $\mathrm{N}$-ray problem, which would seem definitely to solve the question as to the real existence of these rays, is publishe

Prof. E. Mascart a short time ago asked Prof. Blondlot to repeat in his presence, with special precautions, the experiment of the refraction of N-rays in an aluminium prism. Prof. Blondlot agreed. The refraction which on account of the experimental conditions are so wide as not to admit of the accuracy of optical measurements.

The screen, provided with a thin strip of sulphide previously lighted, was mounted on the carriage of a dividing engine, the figure marked by the index on the ruler being recorded each time the observer stopped the carriage at an intensity maximum. The following
figures were obtained by four different observers in the same range:

Blondlo

Gutton

Mascart

381.0
383.4

Average

$\frac{383.4}{382.4}$

$\begin{array}{rrr}387.2 & 391.5 & 398.4 \\ 393.0 & 399.0\end{array}$

$\begin{array}{lll}386.9 & 392.0 & 398.2\end{array}$

In another experiment the dividing engine was so displaced as to have the motion of the carriage occur approximately at right angles to the refracted rays,
readings being taken in alternate directions. At each stoppage on a maximum. Prof. Mascart would read the corresponding division of the index without the knowledge of the observer.

Prof. Blondlot thus obtained:

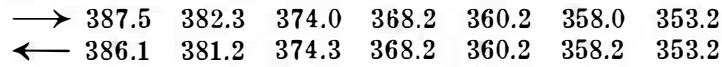

$\begin{array}{lllllll}\text { Average } \overrightarrow{386.8} & \overrightarrow{381.75} & \overrightarrow{374.15} & \overrightarrow{368.2} & \overline{360.2} & \overrightarrow{358.1} & \overrightarrow{353.2}\end{array}$

The Nernst lamp giving off the rays was next extinguished by breaking the circuit, and another set of observations begun on a reduced range to avoid the obtained:

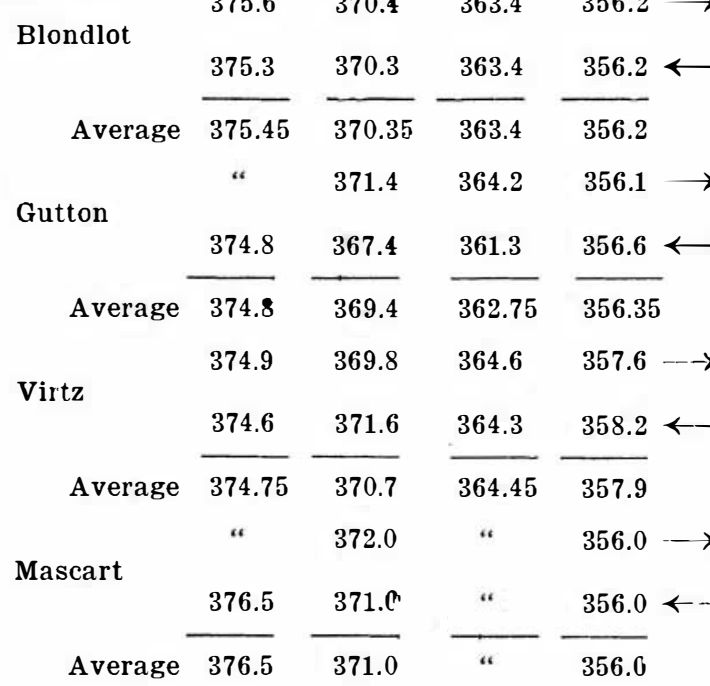

In order to give an idea of the accuracy of these experiments, it may be said that in the latter case the of the scale corresponded to about 4 min. 1 millimeter tions. Prof. Blondlot's pointings were especially accurate, agreeing with each other to less than $1 / 2$ millimeter, apart from two exceptions, while Prof. Mascart's observations, in view of his lesser skill in this special line of work, obviou sly admitted of less accuThe above report was recently presented to the French Academy of Sciences, and at the same meeting Prof. C. Gutton communicated the following on the same problem: If $\mathrm{N}$-rays be made to strike the primary spark of a Hertzian oscillator, the secondary spark decrease in intensity. The action of $\mathrm{N}$-rays is thus shown to alter the electrical phenomenon itself. The action of the primary on the secondary circuit being rendered less energetic by the rays, which reduce the will obviously distane, the inte

This has been evidenced and photographed by $M$. Gutton with the aid of brass electrodes. Brass is in fact an alloy sufficiently hard to take a regular- shape, while the zinc contained therein imparts to the spark the spark is taken at 1-30 to $1-40$ millimeter, the lighting of the plate being chosen so as just to give an appreciable photographic impression. It may be said that a very slight variation in the intensity of lighting in the neighborhood of critical value will result in a considerable variation in the photographic action. Prof. Gutton's memoir contains the detailed descripwell agreeing experiments have shown the intensity 